\title{
Folding of Cantor String
}

\author{
Renu Chugh \\ Department of Mathematics, \\ Maharshi Dayanand \\ University, Rohtak- \\ 124001(INDIA)
}

\author{
Mandeep Kumari \\ Department of Mathematics, \\ Maharshi Dayanand \\ University, Rohtak- \\ 124001(INDIA)
}

\author{
Ashish Kumar \\ Department of Mathematics, \\ RPS Degree College, Balana, \\ Mahendergarh(INDIA)
}

\begin{abstract}
In recent years, folding of various objects have been generated using different approaches. The classical Cantor set is an interesting mathematical construction with links to several areas of analysis and topology. The purpose of this paper is to represent the folding of Cantor string (compliment of Cantor set) using direct folding and folding by cut methods. Moreover, the results governing these types of folding are obtained.
\end{abstract}

\section{Keywords}

Cantor set, Cantor string, Folding methods, Retraction.

\section{INTRODUCTION}

Cantor set is a classical example of perfect subset of the closed interval $[0,1]$, which has the same cardinality as the real line but whose Lebesgue measure is zero[10]. It was discovered in 1875 by Henry John Stephen Smith [33] and first introduced by German mathematician George Cantor (1845-1918) that become known as Cantor ternary set [3-8]. Later on, Devil's and other researchers gave graphical representation of Cantor set in the form of staircases [28-30].

The folding of a manifold was, firstly introduced by Robertson in 1977 [31]. When a sheet of paper is crumpled in the hands and then crushed flat against a desk-top, the pattern so formed is governed by certain simple rules. These rules are generalized to theorems on folding manifolds isometrically into one another which has been examined independently by Robertson [1977] and Sewell [1973]. In 1986, the topological character of the manifolds has been introduced by Robertson et al. [32]. Later on, in a series of papers, Ghoul [15-20], Ghoul et al. [12-14] jointly with others carried further analysis and generalizations of manifolds (see also [24]). Also, for more properties and applications of manifolds in analysis one may refer to $[1,9,20-23,25-27]$. The limit of folding of a manifold is defined in [22].

Lapidus and van Frankenhuijsen [30, 31] introduced the concept of fractal string and established the geometric zeta function, zeros of zeta function, spectra of fractal string and the complex dimension of the fractal string. Further in 2008, Lapidus [32] suggested that fractal string and their quantization may be related to aspects of string theory. In last few decades, M. L. Lapidus, jointly with other researchers generalized and introduced the various properties of fractal string.

In 2012, Attiya [2] studied the folding of hyperbolic manifold using mathematical results. Further, in 2012, the types of retractions of one dimensional manifolds and the isometric and topological folding have been studied by A.E. El-Ahmady et al.[11].

In this paper, we study the folding of Cantor string using two different approaches of folding. The limit of folding of a Cantor string is also discussed. Section 2 deals with some basic definition pertaining to the folding of a manifold, Cantor set and Cantor string. In Section 3, the main result of our study has been presented.

\section{PRELIMINARIES}

Definition 1. Let $M$ be a non-empty (second-countable) Hausdorff topological space such that:

(i) $M$ is the union of open subsets $U \alpha$ and each $U \alpha$ is equipped with a homeomorphism $\mathrm{x} \alpha$ taking $\mathrm{U} \alpha$ to an open set in $\mathrm{Rn}$, i.e.;

$\mathrm{x} \alpha: \mathrm{U} \alpha \rightarrow \mathrm{x} \alpha(\mathrm{U} \alpha) \subset \mathrm{Rn}$

(ii) If $\mathrm{U} \alpha \cap \mathrm{U} \beta=\mathrm{W} \neq \phi$, the sets $\mathrm{x} \alpha-1(\mathrm{~W})$ and $\mathrm{x} \beta-1(\mathrm{~W})$ are open sets in Rn then the overlap map

$$
x_{\beta}^{-1} \circ x_{\alpha}: x_{\alpha}(W) \rightarrow x_{\beta}(W)_{\mathrm{x} \beta} x_{\alpha}^{-1}: \mathrm{x} \alpha(\mathrm{W}) \rightarrow \mathrm{x} \beta(\mathrm{W})
$$

is a smooth map, see Fig.1, where the map $x_{\beta}^{-1} \circ x_{\alpha}$ from an open set in $\mathrm{Rn}$ to $\mathrm{Rn}$ is smooth if all partial derivatives of all orders of each component of the map exist everywhere, where the map is defined.

Each pair $(\mathrm{U} \alpha, \mathrm{x} \alpha)$ is called a chart on $\mathrm{M}$, and the collection A $=\{(\mathrm{U} \alpha, \mathrm{x} \alpha)\}$ of charts is called (smooth) atlas on M. The space $\mathrm{M}$ taken together with the atlas $\mathrm{A}$ will be called a smooth manifold of dimension $\mathrm{n}$ or smooth $\mathrm{n}$-manifold or $\mathrm{C} \infty$ n-manifold. 


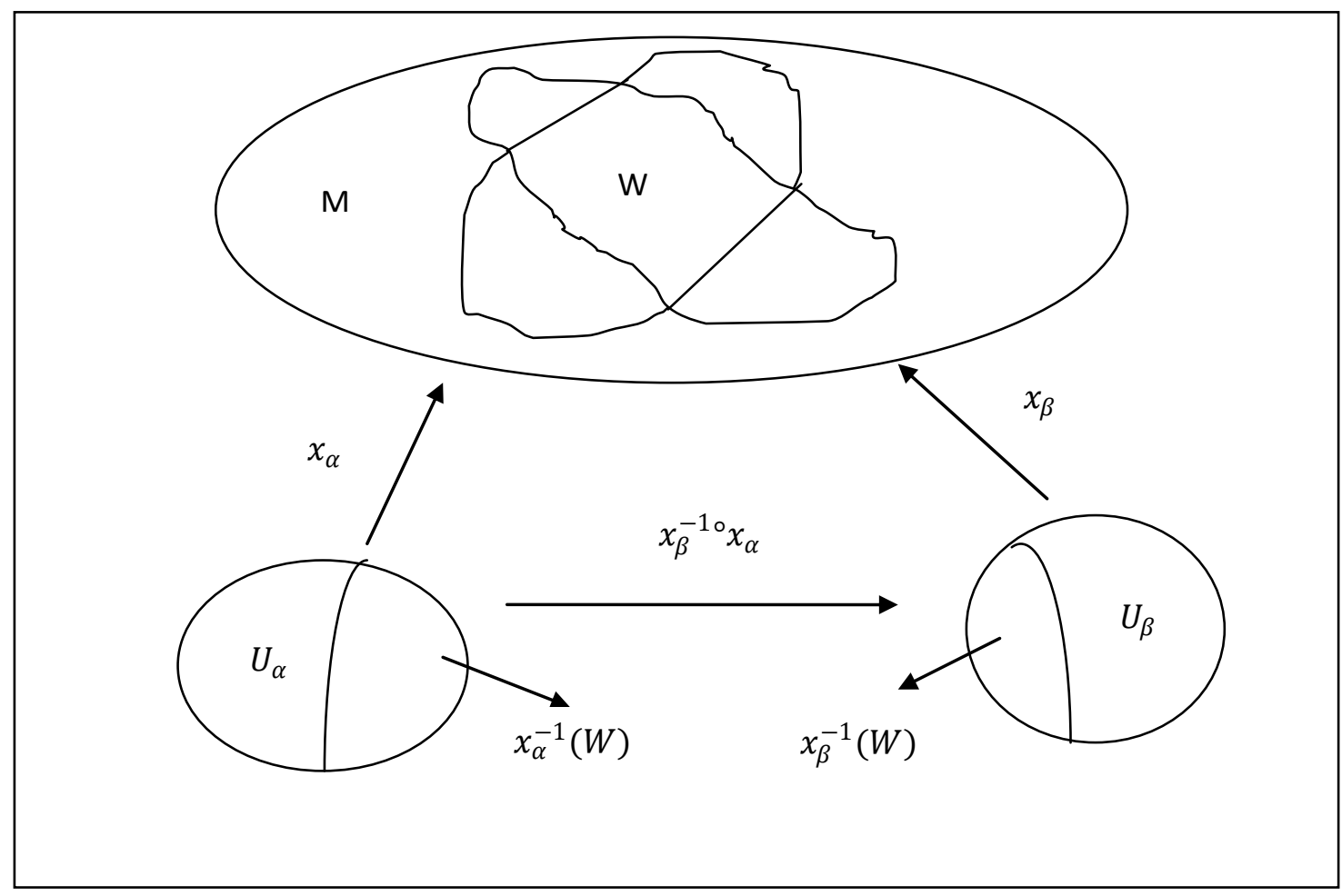

Fig. 1 Smooth mapping

Definition 2. Let $\mathrm{M}$ and $\mathrm{N}$ be two $\mathrm{C} \infty$ - Riemannian manifolds of dimension $\mathrm{m}$ and $\mathrm{n}$ respectively. A map $\mathrm{f}: \mathrm{M} \rightarrow \mathrm{N}$ is said to be an isometric folding of $\mathrm{M}$ into $\mathrm{N}$ if for every piecewise geodesic path $\gamma: \mathrm{I} \rightarrow \mathrm{M}$ the induced path $\mathrm{f}$ o $\gamma: \mathrm{I} \rightarrow \mathrm{N}$ is piecewise geodesic and of the same length as $\gamma$. If $\mathrm{f}$ does not preserve length it is called a topological folding. [34]

Definition 3. A subset A of a topological space is a retract of $\mathrm{X}$ if there exist a continuous map $\mathrm{r}: \mathrm{X} \rightarrow \mathrm{A}$ called a retraction such that $\mathrm{r}(\mathrm{a})=\mathrm{a}$ for any $\mathrm{a} \in \mathrm{A}$.[20]

Definition 4. The Cantor set $\mathrm{C}$ is defined as $\mathrm{C}=\bigcap_{n=1}^{\infty} I_{n}$, where $I_{\mathrm{n}+1}$ is constructed by trisecting $I_{\mathrm{n}}$ and removing the middle third, $\mathrm{I}_{0}$ being the closed real interval $[0,1]$

In 2000, Lapidus and van Frankenhuijsen introduced the concept of fractal strings. They defined it as follows:

Definition 5. A fractal string $\Omega$ is a bounded open subset of the real line $R$. The collection of lengths $\ell j$ of the disjoint intervals is denoted by L. For example, the complement of the Cantor set in the closed unit interval $[0,1]$ is a Cantor string. Moreover, the topological boundary of a Cantor string is the Cantor set $\mathrm{C}$ itself.

Definition 6. The limit of the folding of an $n$-dimentional manifold $\mathrm{M}$ into itself is a manifold $\mathrm{N}$ of dimension $\mathrm{n}-1$. [22]

\section{MAIN RESULTS}

In the year (1879-1884), George Cantor coined few problems and consequences in the field of set theory. One of them was Cantor ternary set a classical example of fractals. Under the constructions of Cantor set, we begin with the closed interval $\mathrm{I} 0=[0,1]$ and divide it into three equal open sub-intervals. And remove the central open interval I1 $=\left(\frac{1}{3}, \frac{2}{3}\right)$ such that

$$
[0,1]-\mathrm{I}_{1}=\left[0, \frac{1}{3}\right] \cup\left[\frac{2}{3}, 1\right]
$$

by repeating the process of removing the middle one third from each closed interval, we can define as

$$
\mathrm{C}=\bigcap_{n=1}^{\infty} I_{n}
$$

where $I_{n+1}$ is constructed as above by trisecting $I_{n}$ and removing the middle third, $\mathrm{I}_{0}$ being the closed interval $[0,1]$.

In this paper, we study the folding of Cantor string. To start the constructions of folding of Cantor string, we take the closed interval $[0,1]$ and divide it into 3 equal subintervals. Fig.2 below shows the representation of folding of Cantor string by using direct method.

To start the construction, initiator $\mathrm{F}_{0}=[0,1]$ is subdivided into three equal subintervals, left(L), $\operatorname{right}(\mathrm{R})$ and middle(M). Drop first and third semi-open intervals $x_{1}=\left[0, \frac{1}{3}\right)$ and $x_{2}=\left(\frac{2}{3}, 1\right]$ such that

$\mathrm{F}_{1}=[0,1]-\left[0, \frac{1}{3}\right) \cup\left(\frac{2}{3}, 1\right]=\left[\frac{1}{3}, \frac{2}{3}\right]$

Again we subdivide the closed interval $F_{1}=\left[\frac{1}{3}, \frac{2}{3}\right]$ into three equal subintervals and remove the first and third semi-open intervals, then we get

$\mathrm{F}_{2}=\left[\frac{1}{3}, \frac{2}{3}\right]-\left[\frac{1}{3}, \frac{4}{9}\right) \cup\left(\frac{5}{9}, \frac{2}{3}\right]=\left[\frac{4}{9}, \frac{5}{9}\right]$

Further, repeating the same process again and again, by removing the semi-open subintervals of first and third position at each step from each closed interval, we obtain a sequence $\left\{F_{k}\right\}_{k=1}^{\infty}$. 
Thus the folding of Cantor string would be the limit $\mathrm{F}$ as the intersection of sets $\mathrm{F}_{\mathrm{k}}$ i.e. $\mathrm{F}=\cap F_{k}$

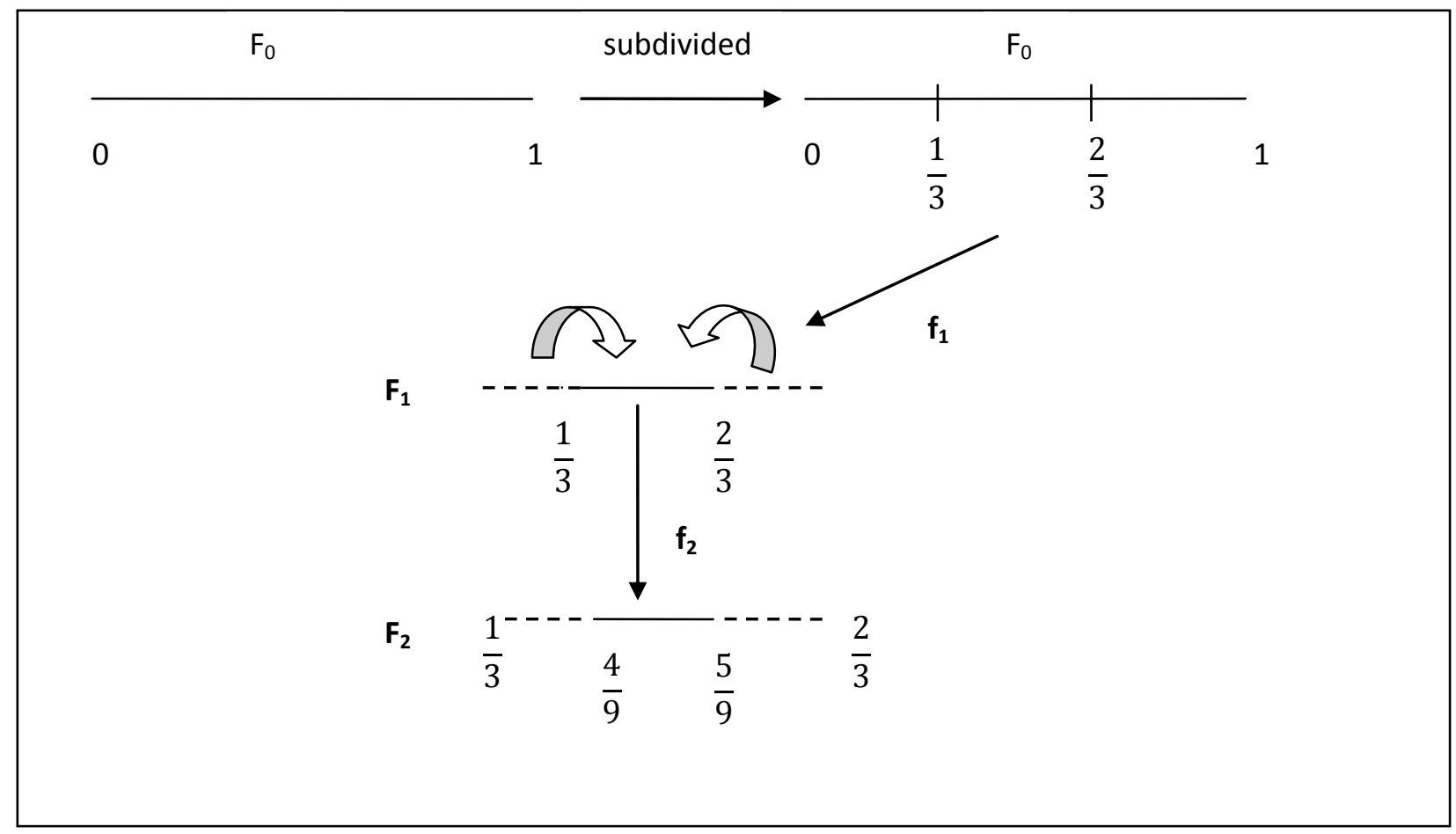

Fig. 2 Folding of Cantor string by direct method

Theorem 3.1 Let $\mathrm{f}$ be the folding map on $[0,1]$ defined by $\mathrm{f}(\mathrm{x})=\frac{x+1}{3}$. Then the Cantor string $\mathrm{F}$ defined above satisfies the inclusion $F_{k} \supseteq F_{k+1}$ for all $k \in\{0,1,2 \ldots\}$.

Proof. In the starting of this section, we study the folding of Cantor set by simply removing the one-third semi-open intervals of first and third step.

Now using the map $\mathrm{f}(\mathrm{x})=\frac{x+1}{3}$, we generate the folding which is quite different from the method mentioned above.

In Fig.3, by using the mapping $\mathrm{f}(\mathrm{x})=\frac{x+1}{3}$ on initiator $[0,1]$, we study the folding of Cantor string in the following way:

First, let $x \in[0,1]$ and then using the map $f$, we get

$$
\begin{aligned}
& \mathrm{f}_{1}\left(\mathrm{~F}_{0}\right)=\mathrm{f}_{1}([0,1])=\left[\frac{1}{3}, \frac{2}{3}\right]=\mathrm{F}_{1} \\
\Rightarrow \quad & \mathrm{F}_{0} \supseteq \mathrm{F}_{1}
\end{aligned}
$$

Now, take $\mathrm{x} \in\left[\frac{1}{3}, \frac{2}{3}\right]$ and then using the mapping $\mathrm{f}$, we get

$$
\begin{aligned}
\mathrm{f}_{2}\left(\mathrm{~F}_{1}\right) & =\mathrm{f}_{2}\left(\left[\frac{1}{3}, \frac{2}{3}\right]\right)=\left[\frac{4}{9}, \frac{5}{9}\right]=\mathrm{F}_{2} \\
\Rightarrow & \mathrm{F}_{1} \supseteq \mathrm{F}_{2}
\end{aligned}
$$

Again, taking $\mathrm{x} \in\left[\frac{4}{9}, \frac{5}{9}\right]$ and then using the mapping $\mathrm{f}$, we get

$$
\begin{array}{rlrl} 
& & \mathrm{f}_{3}\left(\mathrm{~F}_{2}\right)=\mathrm{f}_{2}\left(\left[\frac{4}{9}, \frac{5}{9}\right]\right)=\left[\frac{13}{27}, \frac{14}{27}\right]=\mathrm{F}_{3} \\
\Rightarrow & \mathrm{F}_{2} \supseteq \mathrm{F}_{3}
\end{array}
$$

Fig. 3 shows the geometrical representation of the folding by using a folding map.

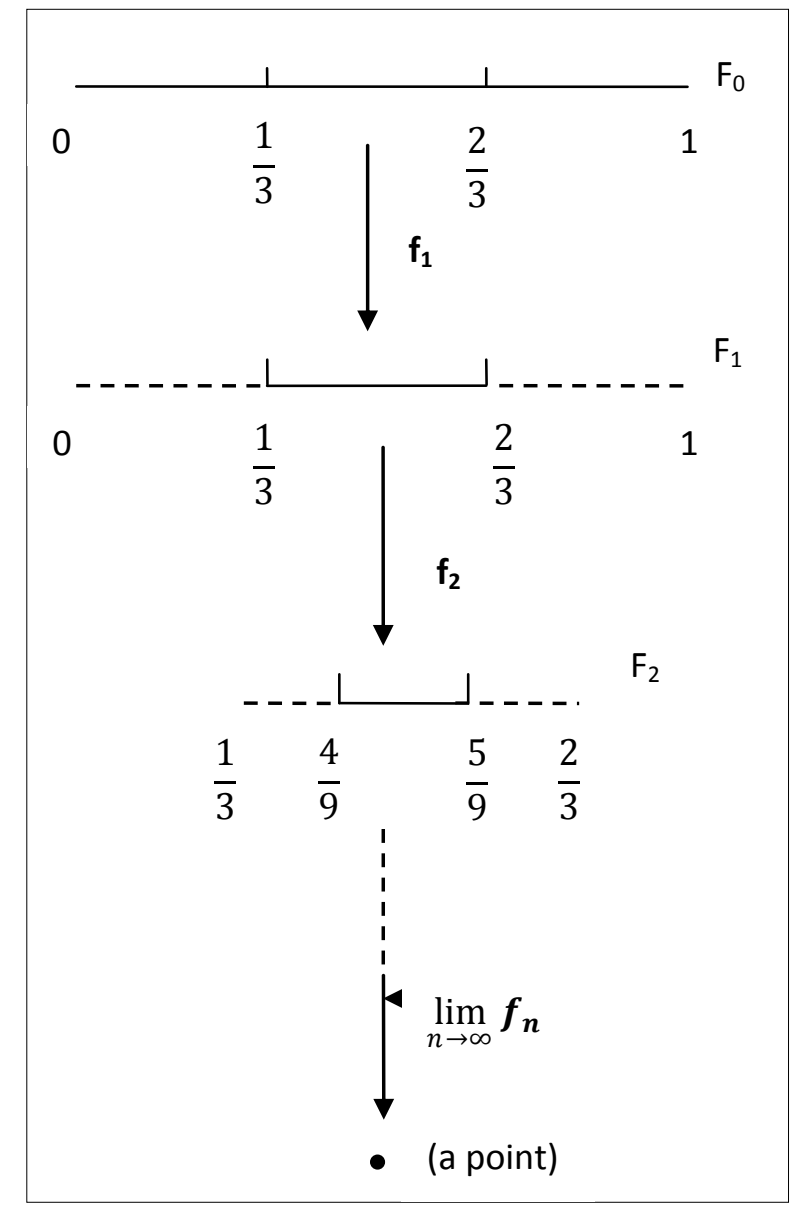

Fig. 3 
Further repeating the same process and substituting the value of previous steps in the mapping $\mathrm{f}(\mathrm{x})=\frac{x+1}{3}$, we get the following table:

Table-1

\begin{tabular}{|c|c|c|}
\hline \multicolumn{2}{|c|}{$\mathrm{f}(\mathrm{x})=\frac{x+1}{3}$} & $\mathrm{~F}_{1}$ \\
\hline $\mathrm{f}_{1}([0,1])$ & {$\left[\frac{1}{3}, \frac{2}{3}\right]$} & $\mathrm{F}_{2}$ \\
\hline $\mathrm{f}_{2}\left(\left[\frac{1}{3}, \frac{2}{3}\right]\right)$ & {$\left[\frac{13}{27}, \frac{14}{27}\right]$} & $\mathrm{F}_{3}$ \\
\hline $\mathrm{f}_{3}\left(\left[\frac{4}{9}, \frac{5}{9}\right]\right)$ & {$\left[\frac{40}{81}, \frac{41}{81}\right]$} & \\
\hline $\mathrm{f}_{4}\left(\left[\frac{13}{27}, \frac{14}{27}\right]\right)$ & {$\left[\frac{121}{243}, \frac{122}{243}\right]$} & $\mathrm{F}_{4}$ \\
\hline $\mathrm{f}_{5}\left(\left[\frac{40}{81}, \frac{41}{81}\right]\right)$ & ------ & $\mathrm{F}_{5}$ \\
\hline------ & {$\left[\frac{\sum_{k=1}^{n} 3^{k-1}}{3^{n}}, \frac{1+\sum_{k=1}^{n} 3^{k-1}}{3^{n}}\right]$} & $\mathrm{F}_{\mathrm{n}}$ \\
\hline $\mathrm{f}_{\mathrm{n}}\left(\mathrm{F}_{\mathrm{n}-1}\right)$ & ----- \\
\hline------- & ------ \\
\hline
\end{tabular}

This implies that the inclusion $F_{k} \supseteq F_{k+1}$ holds for all $k \in\{0,1,2 \ldots\}$. This completes the proof of the theorem.

Theorem 3.2 The limit of folding by cut of Classical Cantor set $[0,1]$ is a 0 -dimensional manifold.

Proof. Here we take closed interval $\mathrm{F}_{0}=[0,1]$ and divided it into 3 equal subintervals.

We remove $\mathrm{p}_{1}=\left[0, \frac{1}{3}\right)$ and $\mathrm{p}_{2}=\left(\frac{2}{3}, 1\right]$ such that

$$
[0,1]-\mathrm{p}_{1} \cup \mathrm{p}_{2}=\left[\frac{1}{3}, \frac{2}{3}\right]=\mathrm{F}_{1}
$$

Next subdivide $F_{1}$ into 3 equal subintervals and then remove two semi open intervals $\mathrm{p}_{3}=\left[\frac{1}{3}, \frac{4}{9}\right)$ and $\mathrm{p}_{4}=\left(\frac{5}{9}, \frac{2}{3}\right]$, we get $\quad \mathrm{F}_{1}-\mathrm{p}_{3} \cup \mathrm{p}_{4}=\left[\frac{4}{9}, \frac{5}{9}\right]=\mathrm{F}_{2}$

In this way we divide the middle closed interval in three equal sub-intervals and then remove the outer two semiclosed intervals. We then find a set of closed intervals, i.e.,

$$
\mathrm{F}=[0,1]-\cup \mathrm{p}_{\mathrm{n}}=\bigcap_{n=1}^{\infty} F_{n}
$$

In each step, we removed $[a, a+w)$ and $(b-w, b]$ intervals from $[\mathrm{a}, \mathrm{b}]$, where $\mathrm{w}=\frac{b-a}{3}$.

Theorem 3.3 The folding of Cantor string is nonempty.

Proof. In the folding of $F_{n}$ to form $F_{n+1}$ leaves a closed interval. For example removing $\left[0, \frac{1}{3}\right)$ and $\left(\frac{2}{3}, 1\right]$ from $[0,1]$ leaves $\left[\frac{1}{3}, \frac{2}{3}\right]$. In fact, since the folding of Cantor string is the infinite intersection of each $F_{n}$, F contains at least one subinterval and is clearly nonempty and infinite.

Theorem 3.4 The folding of Cantor string is uncountable.

Proof. Georg Cantor demonstrated that real numbers cannot be put into one-to-one correspondence with the natural numbers and therefore that the set of real numbers has a greater cardinality than the set of natural numbers. By folding method, we get a nonempty closed subinterval of real numbers which is uncountable.

Theorem 3.5 The limit of folding of Cantor string $[0,1]$ is equal to the limit of retraction.

Proof. Let $\mathrm{r}_{\mathrm{i}}: \mathrm{I}_{\mathrm{i}}=(\mathrm{a}, \mathrm{b}) \rightarrow[\mathrm{a}+\mathrm{w}, \mathrm{b}-\mathrm{w}]$, be the retraction and the limits of folding for $[0,1]$ are given by $\mathrm{f}_{\mathrm{i}}: \mathrm{I}_{\mathrm{i}}=[\mathrm{a}, \mathrm{b}] \rightarrow$ $[\mathrm{a}+\mathrm{w}, \mathrm{b}-\mathrm{w}]$, where $\mathrm{w}=\frac{b-a}{3}$.

$$
\text { Let } \begin{aligned}
r_{1}: C_{l} \rightarrow & C_{2}, C_{2} \subset C_{1} \\
& r_{2}: C_{2} \rightarrow C_{3} \\
& \ldots \ldots \ldots \ldots \\
& r_{n}: C_{n} \rightarrow C_{n+1}
\end{aligned}
$$

Then $r_{i} \equiv f_{i}$, fig. 4 represents that there are homeomorphisms $\mathrm{h}_{\mathrm{i}}$ such that

$$
h_{\mathrm{n}+1} \circ \lim _{n \rightarrow \infty} r_{\mathrm{n}}=\lim _{n \rightarrow \infty} f_{\mathrm{n}} \circ h_{\mathrm{n}+1} .
$$

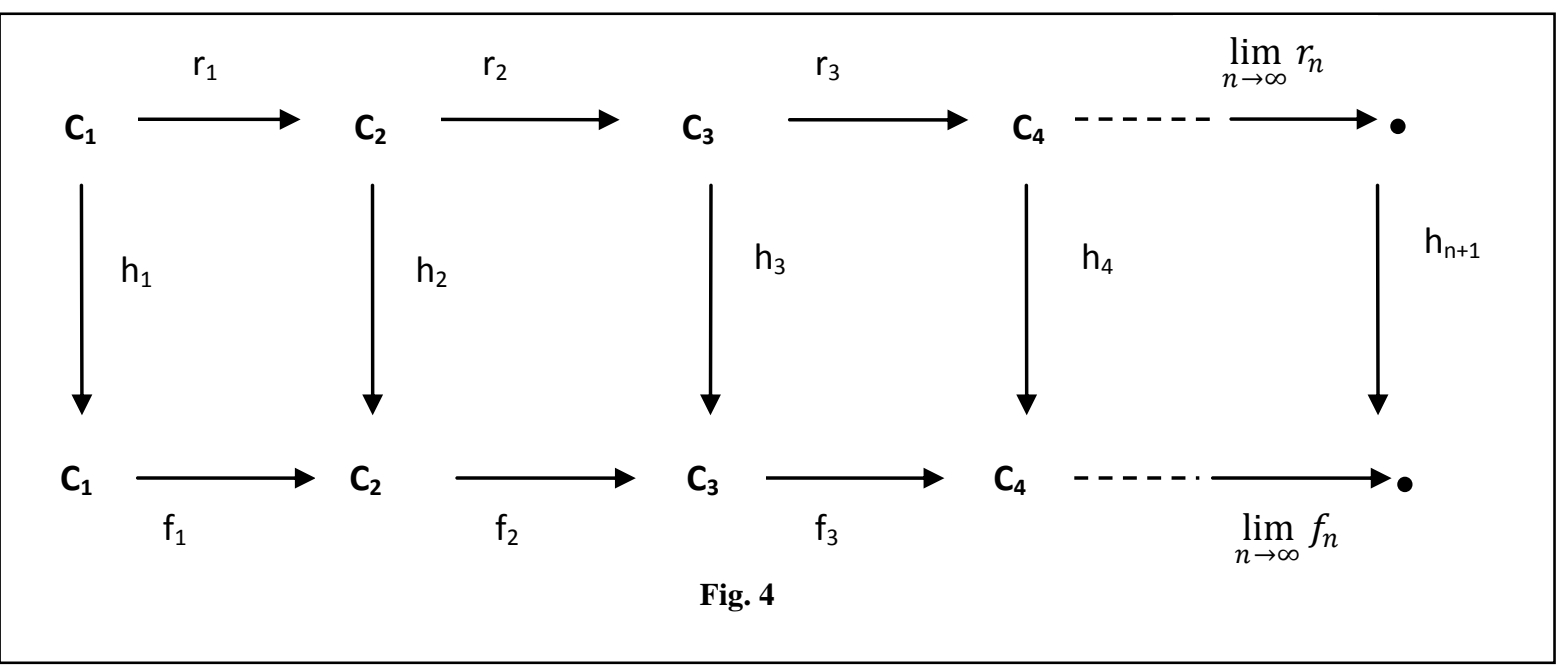




\section{CONCLUSION}

In this paper, different Folding methods have been introduced in the study of Cantor string. Also, the limits of folding and retraction are identical. We have drawn different diagrams to give the description of our approach. Thus, our work is the application of folding in the field of Cantor set theory.

\section{ACKNOWLEDGMENTS}

This Research is supported by the University Grant Commission of India (Grant No. 39-29/2010(SR)).

\section{REFERENCES}

[1] Abu Saleem, M. 2007 Some geometric transformations on manifolds and their algebraic structures, Ph.D. Thesis, University of Tanta, Egypt.

[2] Attiya, H. I. 2012. Folding of Hyperbolic Manifolds, Int. J. Contem. Math. Sciences, Vol.7, N. 36, 1791-1799.

[3] Cantor, G. 1879. Uber unendliche lineare Punktmannichfaltigkeiten, Part 1, Math. Ann. Vol.15, 1-7.

[4] Cantor, G. 1880. Uber unendliche lineare Punktmannichfaltigkeiten, Part 2, Math. Ann. Vol.17, 355-358.

[5] Cantor, G. 1882. Uber unendliche lineare Punktmannichfaltigkeiten, Part 3, Math. Ann. Vol.20, 113-121.

[6] Cantor, G.1883. Uber unendliche lineare Punktmannichfaltigkeiten, Part 4, Math. Ann. Vol.21, 51-58.

[7] Cantor, G. 1883 Uber unendliche lineare Punktmannichfaltigkeiten, Part 5, Math. Ann., Vol. 21, 545-591.

[8] Cantor, G. 1884.Uber unendliche lineare Punktmannichfaltigkeiten, Part 6, Math. Ann. , Vol. 23, 453-488.

[9] DI-Francesco, P. 2000. Folding and coloring problem in mathematics and physics, Bull. Amer. Math. Soc. Vol. 37, 251-307.

[10] Edgar, G. 2008 Measure Topology and Fractal Geometry, Springer Verlag, New York, USA.

[11] El-Ahmady, A. E. and Al- Hazmi, N. S. A. 2012 Retraction of one dimensional Manifold, Appl. Math., Vol. 3, 1135-1143.

[12] El-Ghoul, M., El-Ahmady, A. E. and Rafat, H. 2004. Folding-retraction of chaotic dynamical manifold and the VAK of vacuum fluctuation, Chaos Solitons Fractals, Vol. 20, 209-217.

[13] El-Ghoul, M., El-Ahmady, A. E. and Abu-Saleem, M. 2007. Folding on the Cartesian product of manifolds and their fundamental group, Appl. Sci., Vol. 9, 86-91.

[14] El-Ghoul, M., El-Ahmady, A. E., Rafat, H. and AbuSaleem, M. 2005. The fundamental group of the connected sum of manifolds and their foldings, Chungcheong Math. Soc., Vol. 18, 161-172.

[15] El-Ghoul, M. 1993. Folding of fuzzy graphs and fuzzy spheres, Fuzzy Sets Syst., Vol. 58, 355-363.

[16] El-Ghoul, M. 1985. Folding of manifold, Ph.D Thesis, University of Tanta, Egypt

[17] El-Ghoul, M. 2002. Fractional dimension of a manifold, Chaos Solitons Fractals, Vol.14,77-80.

[18] El-Ghoul, M. 2001. Fractional folding of a manifold, Chaos Solitons Fractals Vol. 12,1019-1023.
[19] El-Ghoul, M., and Shamara, H. M. 1988. Folding of some types of fuzzy manifolds and their retractions, Fuzzy Sets Syst., Vol. 97, 387-391.

[20] El-Ghoul, M. 1998. The deformation retract and topological folding of a manifold, Commun. Fac. Sci. Univ. Ankara Ser. A, 37, 1-4.

[21] El-Ghoul, M. 1995. The deformation retract of the complex projective space and its topological folding, J. Mater Sci., Vol. 30, No. 4, 45-48.

[22] El-Ghoul, M. 1997. The limit of folding of a manifold and its deformation retract, J. Egypt Math. Soc., Vol. 5, No. 2, 133-140.

[23] El-Ghoul, M. Unfolding of graphs and uncertain graph, The Australian Senior Mathematics Journal Sandy Bay 7006 Tasmania Australia (accepted).

[24] El-Ghoul, M. and Zeen El-Deen, M. R. 1999. On some local properties of Fuzzy Manifold and its folding, Le Matematiche, Vol. LIV- Fasc.II, 201-209.

[25] El-Kholy, E. M. and Al-Khusoni, 1991. Folding of CWcomplexes, J. Inst. Math. Comp. Sci.(Math Ser), Vol. 4 No.1, 41-48.

[26] El-Kholy, E. M. 1981. Isometric and topological folding of manifold, Ph. D. Thesis, University of Southampton, UK.

[27] El-Kholy, E.M. and El-Ghoul, M. 1984. Simpilicial folding, J. Fac. Education, Ain ShamsUn., 7 (part. II), 127-139.

[28] Horiguchi, T. and Morita, T. 1984. Devil's staircase in one dimensional mapping, Physica A, Vol.126, No.3 328-348.

[29] Horiguchi, T. and Morita, T. 1984. Fractal dimension related to Devil's staircase for a family of piecewise linear mappings, Physica A, Vol.128, No.1-2, 289-295.

[30] Lapidus, M. L. and van Frankenhuijsen, M. 2000. Fractal Geometry and Number Theory: Complex dimensions of fractal strings and zeros of zeta functions. Birkhh"auser, Boston

[31] Lapidus, M. L. and van Frankenhuijsen, M. 2006. Fractal Geometry, Complex Dimensions and Zeta Functions: Geometry and spectra of fractal strings. Springer, Monographs in Mathematics, Springer-Verlag, New York.

[32] Lapidus, M.L. and Hung, L. 2008. Non-Archimedean Cantor set and string, J Fixed Point Theory Appl., Vol.3, No.1, 181-190.

[33] Rani, M. and Prasad, S. 2010. Superior Cantor sets and superior Devil's staircases, Int. J. Artif. Life Res., Vol.1, No.1, 78-84.

[34] Robertson, S. A. 1977. Isometric folding of Riemannian manifolds, Proc. Roy. Soc Edinburgh, Vol. 77, 275-289.

[35] Robertson, S. A. and El-Kholy, E. M. 1986. Topological folding, Commun. Fac. Sci. Univ. Ank., Series $\mathrm{A}_{1}$, Vol. 35, 101-107.

[36] Smith, H. J. S. 1875. On the integration of discontinuous functions, Proc. Lon. Math. Soc., Vol. 6, No.1, 140-153. 